\title{
Influence des facteurs abiotiques sur la sortie des sédiments de Cyclops vicinus et Chaoborus flavicans dans les zones sub-littorale et profonde d'un lac tempéré eutrophe
}

\author{
C. Rabette $^{1}$ \\ N. Lair ${ }^{2}$
}

Mots-clés : Cyclops vicinus, Copepoda, Chaoborus flavicans, facteurs abiotiques, zone sub-littorale, zone profonde, diapause

\begin{abstract}
A la suite des travaux réalisés sur le lac eutrophe d'Aydat, dans lequel une partie de la communauté planctonique quitte la pleine eau (copépodites 4 de Cyclops vicinus et larves IV de Chaoborus flavicans), nous avons étudié la faune benthique en relation avec les variables abiotiques.

Des séries de prélèvements ont été effectuées en 5 stations réparties de la zone sub-littorale $(-4 \mathrm{~m})$ à la zone centrale $(-14 \mathrm{~m})$ du lac, avec une fréquence mensuelle ou bi-mensuelle de juillet 94 à août 95 . Les échantillons ont été prélevés en triplicats au moyen d'une benne Ekman. Contrairement aux variations progressives de température et d'oxygène, la granulométrie n'est pas significativement différente d'une station à l'autre.

La faune sub-benthique utilise les sédiments selon deux stratégies. Les résultats antérieurs ont montré qu'après avoir accumulé des réserves, les copépodites 4 de Cyclops vicinus s'enfouissent en été dans les sédiments anoxiques et nous avons observé leur émergence lors du brassage automnal. En période estivale, il est connu que les larves IV de Chaoborus flavicans occupent alternativement les eaux oxygénées et les sédiments anoxiques; le fait qu'elles ne supportent pas un milieu désoxygéné en permanence est illustré par leur enfouissement massif, dès que se produit la réoxygénation des eaux profondes. En hiver ces larves demeurent dans les sédiments, jusqu'au printemps suivant quand l'anoxie s'installe à nouveau. Les dates de sortie des sédiments de ces organismes sont fonction de la réoxygénation et de l'augmentation de température de leur environnement.
\end{abstract}

Influence of abiotic factors on the migration from the sediments of Cyclops vicinus and Chaoborus flavicans in the sub-littoral and profundal zone of an eutrophic temperate lake

Keywords : Cyclops vicinus, Copepoda, Chaoborus flavicans, abiotic factors, sub-littoral zone, profundal zone, diapause

Different studies on the eutrophic Lake Aydat have shown that a part of the planktonic community $\left(4^{\text {th }}\right.$ copepodite stage of Cyclops vicinus and $4^{\text {th }}$ instar larvae of Chaoborus flavicans) leaves the open water. In this study the benthic fauna has been related to the environmental variables of this lake.

Triplicate samples were collected monthly or bi-monthly from July 94 to August 95 with an Ekman grab at 5 stations from the sub-littoral $(-4 \mathrm{~m})$ to the central part $(-14 \mathrm{~m})$ of the lake. In contrast to progressive oxygen and temperature variations, granulometry is not significantly different from one station to the other.

The sub-benthic fauna uses the sediments by two strategies. Previous results have shown that after a reserve accumulation, the $4^{\text {th }}$ copepodites burrow during summer into the anoxic sediments and emerge only during the autumnal mixing. During summer, the IVth instar larvae of Chaoborus stay alternately in the oxygenated water and in the anoxic sediments. Their ability to support the permanent anoxia of the deep water is illustrated by their massive burrowing since the reoxygenation occurs. They stay in the sediments until the following spring, when the sediments are again deoxygenated. The timing of their release from the sediments depends on the rise of the oxygen depletion and on the temperature increase of their environment.

1. Université Blaise Pascal, Laboratoire de Biologie Comparée des Protistes, UPRES-A 6023, 63177 Aubière Cedex, France.

2. Université Blaise Pascal, Equipe Hydrosystèmes et Bassins Versants, UPRES-A 6042, 63177 Aubière Cedex, France. 


\section{Introduction}

La majorité des traités de limnologie (Brinkhurst 1974, Hutchinson 1975, Wetzel 1975, Dussart 1982, Goldman \& Horne 1983) rapporte que les lacs présentent, en général, trois zones benthiques : littorale, sublittorale (aussi appelée littori-profonde) et profonde. Ces zones sont, en général, définies à l'aide de critères physiques et morphologiques (mouvement des vagues, complexité de l'habitat, pénétration de la lumière, température, etc.). Bien que la profondeur soit reconnue depuis longtemps comme déterminante de la distribution des organismes benthiques, les études concernant la distribution bathymétrique des organismes benthiques dans les eaux douces stagnantes, ainsi que la vie benthique de la méiofaune, sont très rares (Särkkä 1995).

Classiquement, la distribution de la faune benthique est influencée par de nombreux facteurs : température, oxygénation, quantité de nourriture disponible, prédation ou compétition, etc. (Kajak \& Rybak 1979, Stenson 1980, Moore 1981, Bazzanti \& Seminara 1987, Allison \& Harvey 1988, Mac Kay et al. 1990, Büns \& Ratte 1991, Wyngaard et al. 1991, Giani \& Laville 1995). On peut donc s'attendre à ce que les organismes aient optimisé leur cycle de vie et leur comportement en réponse à tous ces facteurs (Van de Bund \& Groenendijk 1994).

La faune sub-benthique est composée d'organismes vivant au contact des sédiments sans leur être intimement liés et pouvant momentanément devenir pélagiques (Juget et al. 1995). Les variations saisonnières de densité et de biomasse de cette faune ont été surtout étudiées durant la phase pélagique. De plus, ces travaux concernent le plus souvent soit le macrobenthos (Durieu \& Thomas 1995) soit le méiobenthos (Strayer 1985, Santer \& Lampert 1995). Cependant, bien que ces deux composants apparaissent simultanément et à des densités non négligeables dans les écosystèmes d'eau douce, la nature de leurs interactions n'est pás encore très claire (Lodge et al. 1988).

Dans le lac d'Aydat, le quatrième stade copépodite de Cyclops vicinus vicinus (Ulianine 1875) entre en diapause dans les sédiments et partage le même habitat que le quatrième stade larvaire de Chaoborus flavicans (Meigen 1818) (Lair \& El Ghachtoul 1989). Dans un tel contexte, le but de cette étude a donc été de mettre en évidence l'influence des facteurs environnementaux sur la sortie du sédiment de cette faune sub-benthique.

\section{Matériels et méthodes}

Le lac d'Aydat est un lac typiquement eutrophe de la zone tempérée (Lat. $45^{\circ} 39^{\prime} 6 \mathrm{~N}$; Long. $2^{\circ} 29^{\prime} 2 \mathrm{E}$; alt. $837 \mathrm{~m}$; prof. max. $15,5 \mathrm{~m}$; prof. moy. 7,4 ; surface 60 ha). Pour plus de détails sur les caractéristiques physiques, chimiques et biologiques, se reporter à Hartmann et al. (1993).

Les prélèvements ont été réalisés de juillet 94 à août 95, tous les quinze jours aux périodes d'entrée et de sortie de diapause des copépodites 4 de Cyclops vici$n u s$, et tous les mois durant le reste de l'année. Les échantillons ont été récoltés à 6 stations réparties le long d'un transect allant de la zone sub-littorale (- $4 \mathrm{~m})$ à la zone profonde $(-14 \mathrm{~m})$. A chaque station échantillonnée, la température et la teneur en oxygène dissous sont mesurées à l'aide d'un oxy-thermomètre modèle WTW 196. Les échantillons de sédiments sont prélevés à l'aide d'une benne Ekman (Surface : 225 $\mathrm{cm}^{2}$, Volume moyen prélevé : $2,5 \mathrm{l}$ ), échantillonneur le plus couramment utilisé pour la récolte du benthos (e.g. Downing 1984). Trois réplicats ont été prélevés à chaque station et transportés immédiatement au laboratoire. Pour faciliter la séparation des organismes, les échantillons sont tamisés sur un jeu de 4 tamis (vide de maille : $5,2,1$ et $0,45 \mathrm{~mm}$ ) ; les organismes ainsi séparés sont préservés à l'aide d'une solution de formol-sucrose (4-6\%), selon Prepas (1978). Les organismes benthiques sont triés, identifiés et comptés à la loupe binoculaire (Wild M3Z). Les échantillons sont examinés en totalité et les copépodites 4 avec et sans bouclier céphalique sont différenciés. Les densités sont pondérées en fonction de la quantité de sédiment collectée à chaque prélèvement.

Une mesure de la texture granulométrique des sédiments récoltés à chaque station est réalisée par tamisage et les particules sont réparties dans les classes granulométriques suivantes : sables grossiers, sables fins, limons grossiers, limons fins et argiles, couramment utilisées (AFNOR Norme NF X 31-101 \& 31-107).

Afin de mettre en évidence les relations entre les organismes benthiques et les paramètres abiotiques à toutes les profondeurs étudiées, nous avons effectué une Analyse en Composantes Principales (ACP). Ce type d'analyse multivariée est couramment utilisé dans les études relatives aux écosystèmes d'eau douce (Dolédec \& Chessel 1994). Nous avons pris en compte les valeurs de densité obtenues pour chaque triplicat. En accord avec Williams et al. (1968) les valeurs absolues pour chaque échantillon à chaque date et chaque profondeur sont traitées ensemble pour réaliser des corrélations linéaires (test bilatéral). Les différences de 
composition granulométrique entre les stations échantillonnées ont été testées au moyen d'un test non paramétrique : le test-U de Mann-Whitney. Pour la suite, nous avons regroupé les données des stations - 6 et - 8 $\mathrm{m}$, correspondant à la zone sub-littorale, et celles des stations - 10, - 12 et - $14 \mathrm{~m}$, correspondant à la zone profonde.

\section{Résultats}

\subsection{Caractéristiques abiotiques}

Les températures enregistrées au cours de l'été 94 ont toujours été inférieures à celles enregistrées durant l'été 95 (Fig. 1). Les valeurs les plus faibles sont toujours mesurées à la station la plus profonde. La température minimale est enregistrée le 16 janvier $95\left(2,2^{\circ} \mathrm{C}\right)$ à toutes les stations, elle est suivie d'une lente augmentation. La température maximale est atteinte le 9 août 95 à toutes les stations échantillonnées.

Concernant la teneur en oxygène dissous (Fig. 2), le 19 juillet 94 , lors de la première campagne de prélèvements, la désoxygénation est déjà installée tout au long du transect, excepté à la station - $4 \mathrm{~m}$ qui ne sera jamais désoxygénée. Entre fin novembre et mi-février, le fond est progressivement réoxygéné et il le reste jusqu'au début mai. Puis, la désoxygénation s'installe à nouveau et atteint progressivement toutes les stations (excepté - $4 \mathrm{~m}$ ).

Les sédiments des différentes stations échantillonnées sont composés de 27 à $29 \%$ d'argiles et de 47 à $64 \%$ de limons fins (Tableau 1). Les particules les plus grosses représentées par les limons grossiers, les sables fins et les sables grossiers sont relativement importantes seulement aux stations les plus proches de la zone littorale. Le test-U de Mann-Whitney a montré qu'il n'existe aucune différence significative entre les différentes stations échantillonnées. Nous pouvons dire que la zone prospectée offre un habitat très stable.

\subsection{Structure de la communauté en fonction des va- riables abiotiques}

Dans le traitement des données par ACP nous avons conservé les valeurs de chaque triplicat à chacune des stations (soit 240 jeux de données). Les axes F1 et F2 extraient respectivement 50,47 et $10,35 \%$ de l'inertie

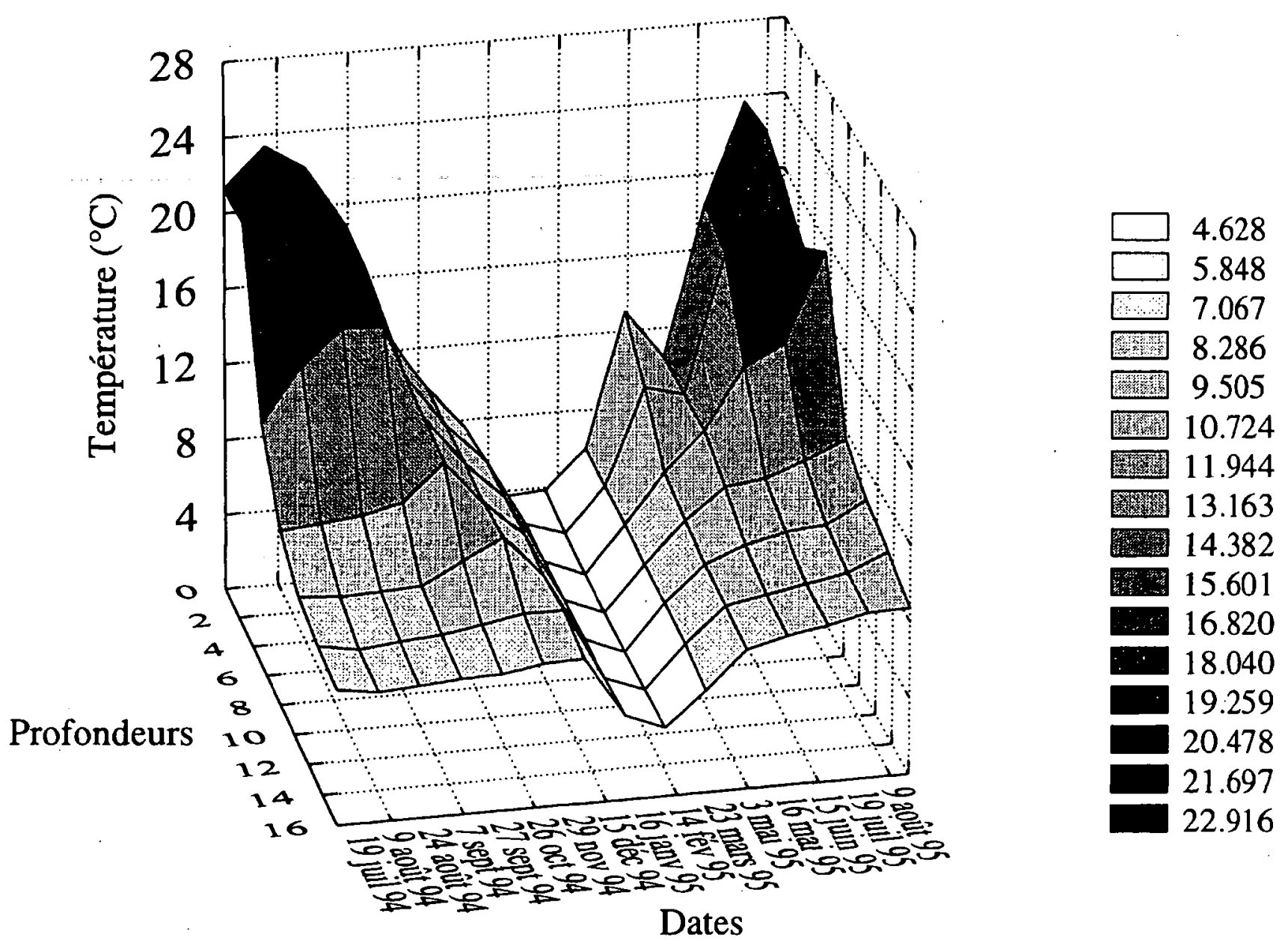

Fig. 1. Variations spatio-temporelles de la température du fond.

Fig. 1. Bottom temperature spatio-temporal variations. 
Tableau 1. Granulométrie des sédiments aux différentes stations prospectées (Pourcentage des différentes classes de taille).

Table 1. Sediment granulometry at the different stations examined (Percent of the different size classes particles).

\begin{tabular}{lrrrrrr}
\hline \multicolumn{1}{c}{ Classes granulométriques } & $4 \mathrm{~m}$ & $6 \mathrm{~m}$ & $8 \mathrm{~m}$ & $10 \mathrm{~m}$ & $12 \mathrm{~m}$ & $14 \mathrm{~m}$ \\
\hline Sables grossiers $(200 \mu \mathrm{m}$ à $2 \mathrm{~mm})$ & 1 & 1 & 1 & 0 & 0 & 0 \\
Sables fins $(50$ à $200 \mu \mathrm{m})$ & 9 & 5 & 4 & 3 & 3 & 2 \\
Limons grossiers $(20 \mathrm{à} 50 \mu \mathrm{m})$ & 12 & 20 & 15 & 12 & 11 & 4 \\
Limons fins $(2 \mathrm{à} 20 \mu \mathrm{m})$ & 49 & 47 & 53 & 55 & 57 & 64 \\
Argiles $(<2 \mu \mathrm{m})$ & 28 & 27 & 27 & 29 & 29 & 29 \\
\hline
\end{tabular}

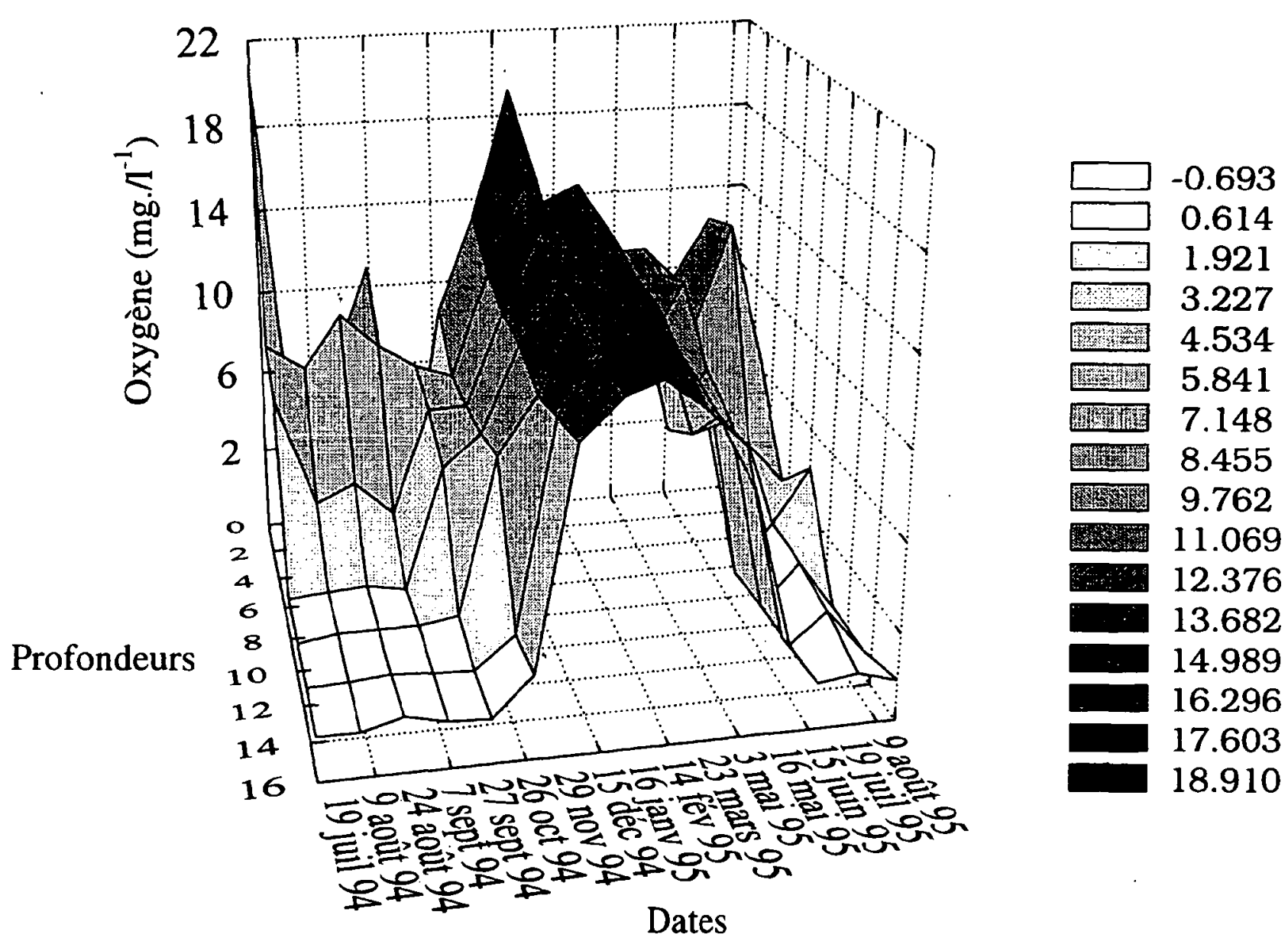

Fig. 2. Variations spatio-temporelles de la teneur en oxygène dissous du fond.

Fig. 2. Bottom oxygen spatio-temporal variations.

totale (Fig. 3). L'axe F1 regroupe les différentes classes granulométriques, il existe une opposition très nette entre les particules les plus grosses (rassemblées dans la partie négative) et les particules les plus fines (regroupées dans la partie positive). L'axe F2 regroupe les organismes : les copépodites 4 de Cyclops vicinus, (avec ou sans bouclier) dans la partie positive, s'opposent aux larves IV de Chaoborus flavicans qui sont associées à la concentration en oxygène dissous dans la partie négative.

\subsection{Distribution spatio-temporelle des organismes}

Le quatrième stade larvaire de Chaoborus flavicans est présent tout au long de l'année dans les sédiments du lac d'Aydat (Fig. 4). Dans la zone littorale sa présence était sporadique à - $4 \mathrm{~m}$, confirmant les observations d'El Ghachtoul (1984). Dans la zone sub-littorale, sa densité varie de 0 ( 29 novembre) à 10865 ind. $\mathrm{m}^{-2}$ (7 septembre 94), pendant la période de stratification.

Dans la zone profonde, le maximum de densité (89 595 ind. $\mathrm{m}^{-2}$ ) est atteint à la fin de l'hiver et le mini- 


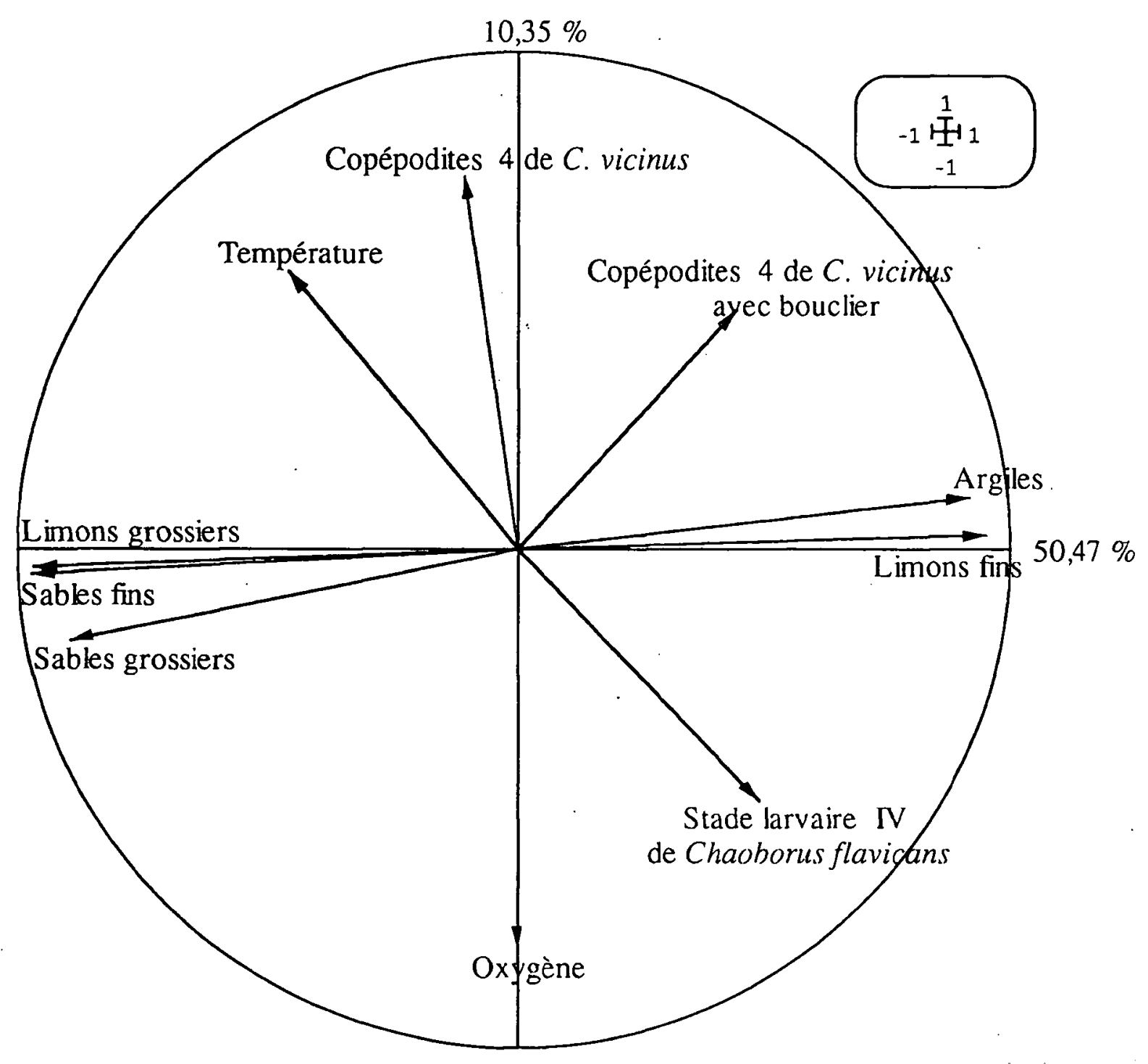

Fig. 3. Position des variables sur le cercle de corrélation défini par les plans des axes F1 et $F 2$.

Fig. 3. Situation of the variables on the correlation circle defined by the planes of the axis F1 and F2.

mum (2240 ind.m-2 ${ }^{-2}$ a été enregistré le 7 septembre 95. Dans cette zone, le quatrième stade larvaire de Chaoborus flavicans est toujours abondant durant la période hivernale et sa densité augmente à la faveur de la réoxygénation des eaux profondes.

Le quatrième stade copépodite de Cyclops vicinus est présent, lui aussi, toute l'année dans les sédiments du lac d'Aydat (Fig. 5). Bien que plus abondant dans la zone profonde, sa répartition au cours de l'année est comparable quelle que soit la zone.

Dans la zone sub-littorale, le maximum de densité est atteint le 29 novembre (11 035 ind.m- ${ }^{-2}$ ). Dans la zone profonde, les individus sont plus nombreux en 95 par rapport à 94 : avec un maximum de 16580 ind. $\mathrm{m}^{-2}$ le 27 septembre. Quelle que soit la zone, à partir du mois de mai et jusqu'à la fin de la période de prélèvement, les copépodites 4 entrent à nouveau progressivement dans les sédiments.
Dès juillet 94 , des individus portant un bouclier céphalique, caractéristique d'une torpeur profonde, ont été observés dans les sédiments. Leur présence toute l'année indique qu'une partie de la population, soit environ $10 \%$ de la totalité des copépodites 4 , reste en diapause dans les sédiments.

\subsection{Influence des variables physiques sur la distri- bution des organismes}

Les variations saisonnières de densité de Cyclops vicinus vicinus et de Chaoborus flavicans sont liées aux variations de température et d'oxygène. En effet, la densité des copépodites 4 en diapause est corrélée positivement à l'augmentation de température et négativement à la concentration en oxygène dissous. A l'inverse, la densité des larves IV de Chaoborus flavicans est corrélée positivement à la concentration en oxygène dissous, mais uniquement dans la zone profonde car 
Tableau 2. Corrélations entre Cyclops vicinus, Chaoborus flavicans et les paramètres abiotiques ( $\mathrm{r}=$ coefficient de corrélation de Pearson ; $\mathrm{P}=$ probabilité vraie).

Table 2. Correlations between Cyclops vicinus, Chaoborus flavicans and the abiotic parametres $(\mathrm{r}=$ Pearson's coefficient, $\mathbf{P}=$ real probability)

\begin{tabular}{cc}
\hline Correlations positives & Correlations négatives \\
\hline $\begin{array}{c}\text { C. vicinus copépodites } 4-\text { Température } \\
(\mathrm{r}=0,06657 ; \mathrm{P}=0,005)\end{array}$ & $\begin{array}{c}\text { C. vicinus copépodites } 4 \text { - Oxygènes } \\
(\mathrm{r}=0,6423 ; \mathrm{P}=0,007)\end{array}$ \\
$\begin{array}{c}\text { C. flavicans larves } \mathrm{IV}-\text { Oxygène } \\
(\mathrm{r}=0,8243 ; \mathrm{P}<0,001)\end{array}$ & $\begin{array}{c}\text { C. flavicans larves } \mathrm{IV}-\text { C. vicinus copépodites } 4 \\
(\mathrm{r}=0,5140 ; \mathrm{P}=0,042)\end{array}$ \\
\hline
\end{tabular}

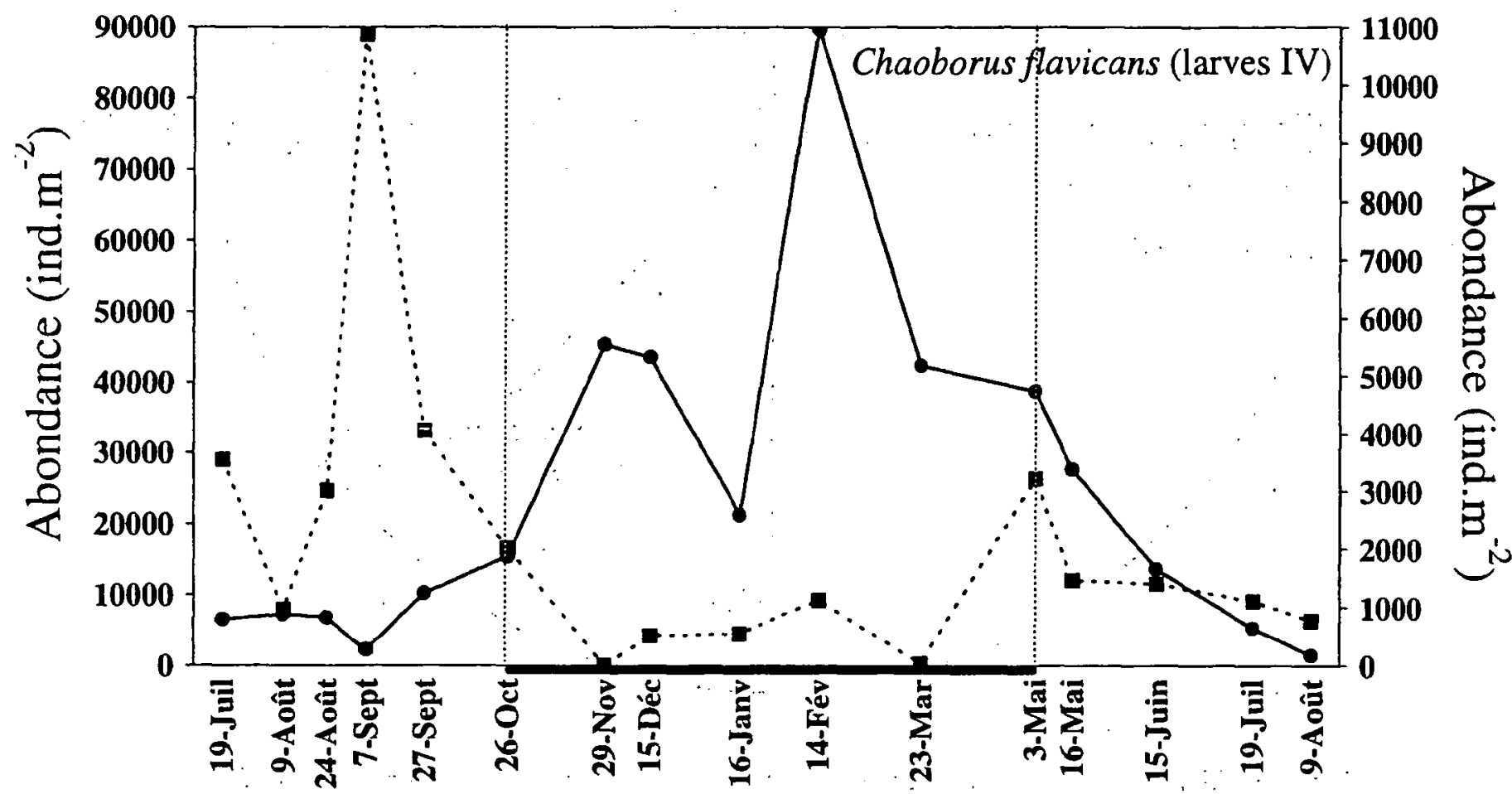

Fig. 4. Variations spatio-temporelles de densité (ind.m-2) des larves IV de Chaoborus flavicans. La zone profonde est représentée par un trait plein (échelle de gauche) et la zone sub-littorale par un trait pointillé (échelle de droite).

Fig. 4. Spatio-temporal density variations of the fourth instar larvae of Chaoborus flavicans. The profundal zone is represented by a full line (left scale) and the sub-littoral zone by a dotted line (right scale).

ce taxon est très peu représenté dans la zone sub-littorale (Tableau 2).

\section{Discussion}

L'absence de différence significative de la texture granulométrique des sédiments le long du transect étudié indique que les variations de répartition spatiale des organismes de la zone sub-littorale à la zone profonde sont indépendantes de la granulométrie.

Comme dans les travaux antérieurs réalisés au lac d'Aydat (Lair \& El Ghachtoul 1989, Lair 1992), au cours des années 94 et 95 nous avons observé que l'entrée des copépodites 4 de $C$. vicinus dans les sédiments se fait lors de l'augmentation de température des sédi- ments et du début de leur désoxygénation. Leur retour en pleine eau est lié au brassage. Il semble donc clairement établi que les facteurs influençant la sortie de diapause des copépodites sont de nature physique.

En outre, une partie (10\% en moyenne) de la population de $C$. vicinus en diapause reste dans les sédiments tout au long de l'année. Dans la revue de ses travaux sur la diapause, Elgmork (1996) a démontré que sa durée est liée à la plus ou moins grande torpeur des organismes. De plus, cet auteur indique que dans un lac du sud de la Norvège (Pond $\mathrm{A}$ ) des individus de $\mathrm{Cy}$ clops strenuus provenant de la même cohorte peuvent se trouver dans un état de torpeur très différent, ce qui doit être également le cas dans le lac d'Aydat. 

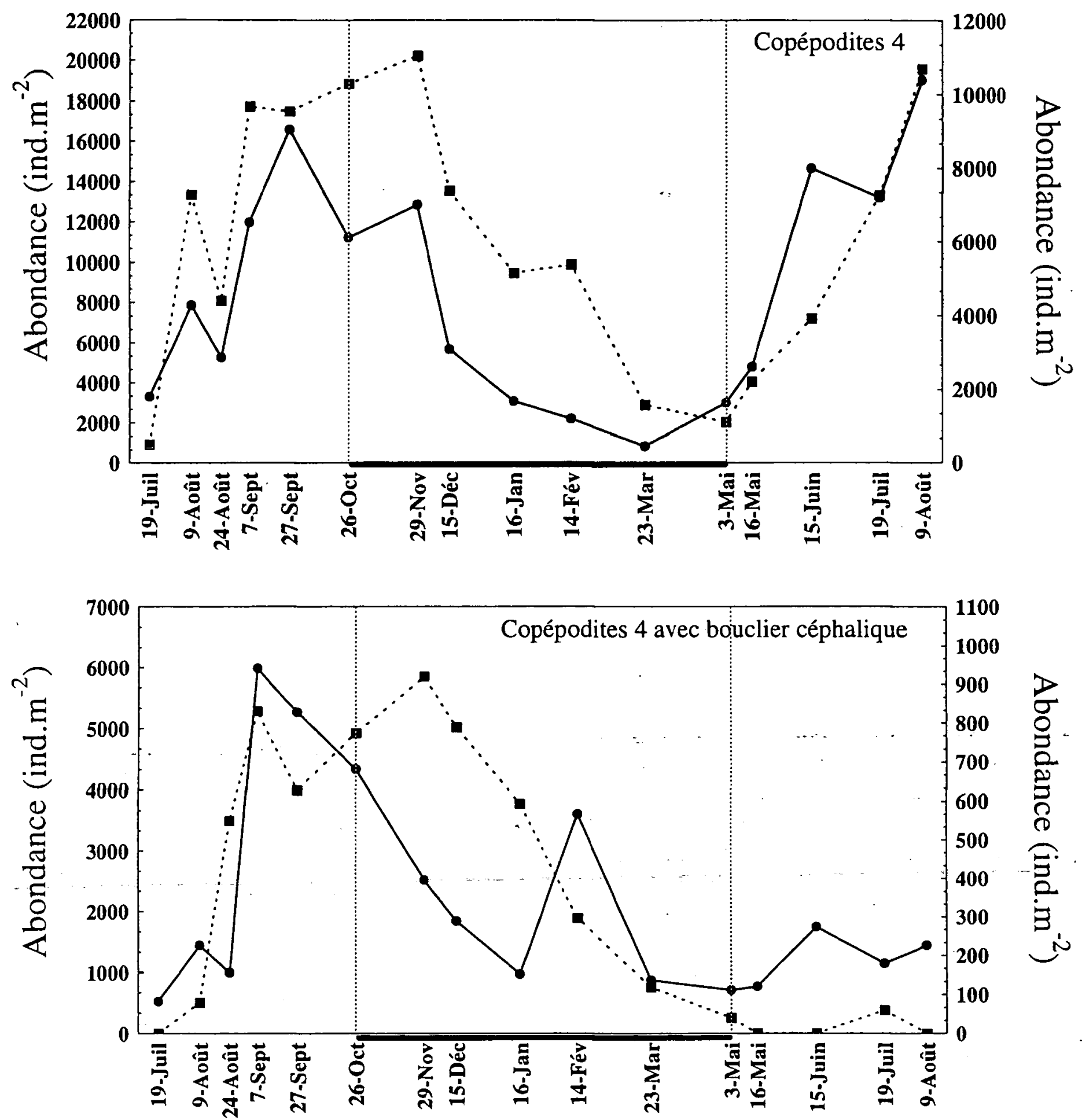

Fig. 5. Variations spatio-temporelles de densité (ind.m-2) des copépodites 4 de Cyclops vicinus. La zone profonde est représentée par un trait plein (échelle de gauche) et la zone sub-littorale par un trait pointillé (échelle de droite). Le trait noir sur l'axe des abscisses délimite la période d'homothermie.

Fig. 5. Spatio-temporal density variations of the fourth copepodite stage of Cyclops vicinus. The profundal is represented by a full line (left scale) and the sub-littoral zone by a dotted line (right scale). The black line on the $\mathrm{x}$ axis corresponds to the homothermic period.

Les larves IV de $C$. flavicans sont des habitants très communs des zones profondes des lacs durant la journée (LaRow 1969). Ces organismes sont capables d'échapper à l'anoxie permanente en réalisant des migrations nocturnes vers l'épilimnion oxygéné et c'est durant ces phases de migration que les larves se nourrissent (e.g. Walter 1985). De telles migrations ont été signalées dans le lac d'Aydat au cours des années 87 et 88 (Gerdeaux et al. 1989, Taleb et al. 1993).

Dans ce lac nous avons observé que les larves IV de C. flavicans sont plus abondantes en hiver et au début du printemps, après le brassage qui induit la réoxygénation du fond. Elles retournent ensuite en pleine eau à la faveur de l'augmentation de température. La densité 
de ces larves est positivement corrélée à la concentration en oxygène dans la zone profonde du lac. Nos résultats nous permettent de penser que ces larves ne peuvent pas vivre dans un environnement complètement désoxygéné.

\section{Conclusion}

Durant l'année, une partie de la faune planctonique cesse de participer à la production de l'écosystème pélagique et va occuper les sédiments fins du lac d'Aydat. Les larves IV de Chaoborus flavicans et les copépodites 4 de Cyclops vicinus occupent en alternance l'espace benthique comme l'indique la corrélation négative qui existe entre leurs abondances respectives. Les différences de répartition de cette faune sub-benthique sont inhérentes à leurs exigences très contrastées par rapport aux variables physiques. Nous retiendrons que la sortie des sédiments de $C$. vicinus et $C$. flavicans paraît réglée par des facteurs physiques.

Il est classiquement reconnu que chez les insectes, les stades de dormance représentent une phase d'économie d'énergie dans la vie de l'espèce (Marshaal et al. 1997), et nous nous sommes interrogées sur la finalité du maintien des larves de Chaoborus dans les sédiments durant l'hiver. Une partie de la population de ce diptère, dont le cycle de vie se déroule dans trois milieux différents, se trouve, à partir de l'automne, dans un habitat composé de sédiments fins où son activité de prédateur est, à l'évidence, non opérationnelle. Tout se passe comme si elle ne trouvait pas en pleine eau les conditions optimales de température et l'énergie suffisante pour atteindre son stade nymphal avant l'hiver. Au printemps, lorsque le plancton se développe à nouveau (Sommer et al. 1988), l'augmentation de température et la réoxygénation du milieu déclenchent son retour en zone pélagique. Toutes les conditions se trouvent alors réunies pour que ses métamorphoses se poursuivent.

Au contraire, les Cyclops quittent la pleine eau au milieu de l'été, pour gagner un habitat désoxygéné, après s'être constitué des réserves (Lair \& El Ghachtoul 1989). A cette période, la température est favoráble à la production planctonique. Tout laisse à penser, comme l'a écrit Neill (1990), qu'il s'agirait bien dans ce cas d'un échappement à la prédation. En effet, nos résultats montrent que leur retour en pleine eau, en période hivernale, s'effectue de surcroît, lorsque les larves de Chaoborus, prédateurs planctoniques particulièrement voraces, se trouvent enfouies dans les sédiments.
Concernant le déclenchement de l'entrée en diapause, seuls les résultats apportés par l'étude des organismes vivant en pleine eau et, en particulier, l'état de la nourriture disponible et le comportement des prédateurs, notamment durant la période précédant l'entrée en diapause des Cyclops nous permettront de conclure (étude en cours).

\section{Travaux cités}

AFNOR 1990. - Normes X 31-101 \& X 31-107. In : Association française de normalisation (ed.), Recueil des normes Françaises. Paris : 101-107.

Allison W.R. \& Harvey H.H. 1988. - Composition, distribution and standing crop of the benthos in a shallow lake. Verh. Internat Verein. Limnol., 23 : 241-245.

Bazzanti M. \& Seminara M. 1987. - Profundal macrobenthos in a polluted lake. Depth distribution and its relationship with biological indices for water quality assessment. Acta Oecologica / Oecol. Applic., 8 : 15-26.

Brinkhurst R.O. 1974. - The benthos of lakes. McMillan Press Ltd, London and Basingstoke : $190 \mathrm{p}$.

Büns M. \& Ratte H.T. 1991. - The combined effect of temperature and food consumption on body weight, egg production and developmental time in Chaoborus crystallinus De Geer (Diptera: Chaoboridae). Some new evidence for the adaptative value of vertical migration. Oecologia, $88: 470-476$.

Dolédec S. \& Chessel D. 1994. - Co-inertia analysis : an alternative method for studying species-environment relationships. Freshwater Biol., $31: 277-294$.

Downing J.A. 1984. - Sampling the benthos of standing waters. In Downing J.A. \& Rigler F.H.(ed.), A manual on methods for the assessment of secondary productivity in fresh waters. Blackwell Sci. Publ., Oxford : 501 p.

Durieu M. \& Thomas A. 1995. - Le régime alimentaire des stades larvaires de Chaoborus flavicans (Diptera : Chaoboridae) dans une lagune d'épuration des eaux usées du Sud-Ouest de la France. Annls Limnol., 31 (4) : 263-275.

Dussart B. 1966. - Limnologie. L'étude des eaux continentales. Gauthier-Villars ed., Paris : $678 \mathrm{p}$.

El Ghachtoul Y. 1984. - Contribution à l'étude du zoobenthos d'un lac eutrophe : le lac d'Aydat. D.E.A. Univ. B. Pascal, ClermontFerrand : $24 \mathrm{p}$.

Elgmork K. 1996. - Variation in torpidity of diapause in freshwater cyclopoid copepods. Hydrobiologia, 320:63-70.

Gerdeaux D., Guillard J. \& Jamet J.L. 1989. — Abundance estimation of Chaoborus larvae and fish in Lake Aydat by echo-integration. Proc. I.O.A., 11 : 231-238.

Giani N. \& Laville H. 1995. - Réseau trophique benthique. In Pourriot \& Meybeck (eds.), Limnologie générale. Masson, Paris : $956 \mathrm{p}$.

Goldman C.R. \& Horne A.J. 1983. - Limnology. Mc Graw-Hill Book Company, New-York : $464 \mathrm{p}$

Hartmann H.J., Taleb H., Aleya L. \& Lair N. 1993. - Predation on ciliates by suspension feeding calanoid copepod. Can. J. Fish. Aquat. Sci., 50: 1382-1393.

Hutchinson G.E. 1975. - A treatise on limnology. II : Introduction to lake biology and limnoplankton. Wiley \& Sons (ed.) New York : $660 \mathrm{p}$.

Juget J., Lafont M., Mouthon J. \& Gerdeaux D. 1995. - Structure des communautés benthiques et pisciaires. In Pourriot \& Meybeck (eds.), Limnologie générale. Masson, Paris : 956 p.

Kajak Z. \& Rybak J. 1979. - The feeding of Chaoborus flavicans Meigen (Diptera; Chaoboridae) and its predation on lake zooplankton. Int. Revue Ges. Hydrobiol., 64 : 361-378. 
Lair N. 1992. - Daytime grazing and assimilation rates of planktonic copepods Acanthodiaptomus denticornis and Cyclops vicinus vicinus. Comparison of spatial and resource utilisation by rotifers ans cladoceran communities in a eutrophic lake. Hydrobiologia, $231: 107-117$

Lair N. \& El Ghachtoul Y. 1989. - La diapause de Cyclops vicinus vicinus (Ulianine, 1875) dans un lac eutrophe du Massif-Central Français. SITE Atti, 7 : 289-295.

LaRow E.J. 1969. - A persistant diurnal rhythm in Chaoborus larvae; II : ecological significance. Limnol. Oceanogr., 14 : 213-218.

Lodge D.M., Barko J.W., Strayer D., Melack J.M., Mittelbach G.G., Howarth R.W., Menge B. \& Titus J.E. 1988. - Spatial heterogeneity and habitat interactions in lake communities. In :Carpenter S.R (ed.), Complex interactions in lake communities. SpringerVerlag, New-York : 181-208.

Mac Kay N.A., Carpenter S.R., Sorano P.A. \& Vanni M.J. 1990. The impact of two Chaoborus species on a zooplankton community. Can. J. Zool., 68 : 981-985.

Marshaal M., Caussanel C. \& Matile L. 1997. — Les Insectes. La Recherche, 292 : 84-87.

Moore J.W. 1981. - Factors influencing the species composition, distribution and abundance of benthic invertebrates in the profundal zone of a eutrophic northern lake. Hydrobiologia, 83 : 505 510.

Neill W.E. 1990. - Induced vertical migration in copepods as a defense against invertebrate predation. Nature, $345: 524-526$.

Prepas E. 1978. - Sugar frosted Daphnia an improved fixation technique for Cladocera. Limnol. Oceanogr., $23: 557-559$.

Santer B. \& Lampert W. 1995. - Summer diapause in cyclopoid copepods: adaptative response to a food bottleneck ? J. Anim. Ecol., 64 : 600-613.
Särkkä J. 1995. - Profundal meiofauna in two large lakes: Influence of pollution and bathymetric differences. Arch. Hydrobiol., 132 : 453-493.

Sommer U., Gliwicz Z.M., Lampert W. \& Duncan A. 1988. - The «PEG « model of seasonal succession of planktonic events in fresh waters. Arch. Hydrobiol., $16: 433-471$.

Stenson J.A.E. 1980. - Predation pressure from fish on two Chaoborus species as related to their visibility. In : Kerfoot W.C. (ed.), Evolution and ecology of zooplankton communities. Univ. Press. New England, Hanover : 411-417.

Strayer D. 1985. - The benthic micrometazoans of Mirror Lake, New Hampshire. Arch. Hydrobiol. Suppl., 72 : 287-426.

Taleb H., Lair N., Reyes-Marchant P. \& Jamet J.L. 1993. Observations on vertical migrations of zooplankton at four different stations of a small, eutrophic, temperate zone lake, in relation to their predators. Arch. Hydrobiol. Beih., $39: 199-216$.

Van de Bund W.J. \& Groenendijk D. 1994. - Seasonal dynamics and burrowing of littoral chironomid larvae in relation to competition and predation. Arch. Hydrobiol., $132: 213-225$.

Walter R. A. 1985. - Benthic macroinvertebrates. In Lickens G.E. (ed.) : An ecosystem approach to aquatic ecology: Mirror Lake and its environment. Chapter V/A/6 Springer, New Hampshire : $516 \mathrm{p}$.

Wetzel R.G. 1975. - Limnology. Sanders ed., Philadelphia : 743 p.

Williams W.T., Lance G.N., Webb L.J., Tracey J.G. \& Dale M.B. 1968. - Studies in the numerical analysis of complex rainforest communities. III. The analysis of successional data. J. Ecol., 57 : 515-535.

Wyngaard G.A., Taylor B.E. \& Mahoney D.L. 1991. - Emergence and dynamics of cyclopoid copepods in an unpredictable environment. Freshwater. Biol., 25 : 219-232. 